found in sunshine, showed that cells in culture activate signalling mechanisms termed the 'UV response' which involve pathways running from a receptor at the cell surface, through the Ras and Raf proteins, to the gene-transcription factors AP-1 and NF-кB (ref. 5). Below the lethal dose, fibroblasts, keratinocytes and melanocytes irradiated with ultraviolet-B (UVB) increase expression of p53 and p21; cellular consequences are cell-cycle arrest and programmed cell death ${ }^{6,7}$.

Stunningly, the new results ${ }^{2}$ - among the first such data from intact human skin - reveal that several protein-degrading enzymes (proteases) are induced by UVB exposures far below the lethal dose, even at a tenth to a hundredth of that required to give a minimally detectable sunburn. These exposures correspond to 2-3 minutes of summer sunshine.

The authors first found 6-60-fold induction of the messenger RNAs for interstitial collagenase, stromelysin and $92 \mathrm{~K}$ gelatinase, enzymes that degrade collagen and elastin during wound repair. The corresponding protease activities were also increased. A $72 \mathrm{~K}$ gelatinase was not induced. Because the three induced genes had a recognition site for the AP-1 transcription factor, which can regulate them in vitro, the authors then used gel-shift assays to show that low UVB doses induce AP-1 in skin. UVB also induced $\mathrm{NF}-\kappa \mathrm{B}$, an in vitro regulator of the $92 \mathrm{~K}$ gelatinase. Inhibitors of AP-1 induction largely blocked induction of these matrix-degrading enzymes.

Because reduced dermal collagen and abnormal elastin are prominent features of sun-damaged skin, the authors suggest that chronic exposure to sunlight slowly degrades the matrix proteins of the dermis, leading to wrinkles and elastosis. If so, prevention of photoageing seems a reachable goal. The inhibitors of AP-1 induction used by Fisher $e t$ al., retinoic acid and a glucocorticoid, are in fact already used as drugs. Retinoic acid, administered under medical supervision, has been shown to reverse unwanted changes in photoaged and normally aged skin ${ }^{1,8}$. The degree to which protease induction is inhibited may predict an individual patient's response to longterm retinoid treatment. Induction itself may predict individual vulnerability to sundamage. Retinoic acid also ameliorates skin cancers and precancers ${ }^{9}$, so low doses of UV may have similar effects on genes regulated by AP-1 and NF- $\mathrm{NB}$ relevant to epidermal cancer. These treatments will be selective for particular UV-induced signal transduction pathways, because Fisher et al. found that inhibiting AP-1 induction did not inhibit sunburn's skin reddening. Others have found that inhibitors of reddening do not inhibit programmed cell death or suppression of the immune system.

But we need to pause here. How, experimentally, can one confirm the tissue-level effect of a specific molecular event that in vivo recurs over a timescale (T) of years? Degradation of collagen and elastin by proteases might well be a beneficial repair response, in which tissue remodelling removes damaged matrix proteins. The side-effects of retinoic acid, at least in current animal models, make it awkward to interpret long-term treatment of UV-irradiated skin, so it will be difficult to isolate causes.

Molecular biologists have had the luxury of almost black-and-white experiments, but data such as those of Fisher et al. now force us to confront long-term, low-dose effects. A similar situation occurs in the origin of skin precancers, which appear to exist in equilibrium between clonal expansion and regression of cells, with sunlight favouring expansion ${ }^{10}$. Whereas the initiated cell is created by a genetic change that is easy to measure, clinical manifestation as a precancer requires the chronic and repetitive forces to be played out.

The challenge will be to find tools for a more sophisticated era, just as geneticists have begun to tackle the subtlety of multigenic diseases by combining their DNA probes with mathematics originally developed for quantitative traits such as body height ${ }^{11}$. The new weapons might include long-term studies on mice with specific gene knockouts; or analysing as a multigenic disease the population differences in patterns of tissue senescence. To shortcircuit the long time spans, comparative evolutionary models have been used to identify correlates of lifespan in a series of mammalian species. Constructing transgenic mice in both long- and short-lived species may be the ultimate approach.

Whatever the tools, they are likely to be of general importance - it seems inescapable that ageing, like precancerous conditions and perhaps atherosclerosis and diabetes ${ }^{12}$, reflects the summation of small but recurrent assaults on the cells of the body. Unlike Godot, $\mathrm{G}_{0} \mathrm{D}_{0} \mathrm{~T}$ eventually arrives.

Douglas E. Brash is in the Departments of Therapeutic Radiology and Genetics, Yale University School of Medicine, New Haven, Connecticut 06510, USA. Barbara A. Gilchrest is in the Department of Dermatology, Boston University School of Medicine, Boston, Massachusetts 02118, USA.

1. Gilchrest, B. A. (ed.) Photodamage (Blackwell Science, Cambridge, MA, 1995

Fisher, G. J. et al. Nature 379, 335-339 (1996)

. Noda, A. et al. Exp. Cell Res. 211, 90-98 (1994).

4. Allsopp, R. C. et al. Proc. natn. Acad. Sci. U.S.A. 89 10114-10118 (1992).

5. Herrlich P et al Adv Enz Reg 34, 381-395 (1994).

6. Liu, M. \& Pelling, J. C. Oncogene 10, 1955-1960 (1995)

7. Medrano, E. E. et al. Cancer Res. 55, 4047-4052 (1995).

8. Kligman, A. M., Dogadkina, D. \& Lavker, R. M. J. Am. Acad. Dermatol. 29, 25-33 (1993).

9. Alirezai, M. et al. J. Am. Acad. Dermatol. 30, 447-451 (1994).

10. Ziegler A et al Nature 372, 773-776 (1994)

11. Lander. E. S. \& Schork, N. J. Science 265. 2037-2048 (1994).

12. Trosko, J. E. \& Chang, C. C. Med. Hypoth. 6, 455-468 (1980).

\section{Tuned heat}

ALmost every engineering component is tense with locked-in stresses. Casting, stamping, moulding, machining, all leave the product rather like a wound-up spring. As a result, everything slowly creeps, even cast iron. The main casting of a lathe must be left for at least a year before final machining; otherwise it will creep in service. Big lasers are often mounted on slabs of natural granite, which has had millions of years to relax its casting stresses.

Another way to relax a material is annealing: heating it up and letting it cool slowly. It shakes down the atomic lattice very inefficiently, and can also introduce contractile stresses when it is withdrawn. Heat, of course, is the random battering of a solid lattice by thermal phonons of all energies. What is needed, says Daedalus, is a sort of 'tuned heat': a blizzard of monochromatic phonons all of the same energy. They would be tuned to just that energy needed to mobilize the lattice defects which lock in the stress. Bathed in the warm glow of a correctly tuned heat, any component would relax completely and anneal perfectly.

So DREADCO physicists are exploiting modern cathode-ray tube and mass spectrometer technology to build a novel electron gun. It will fire electrons with a precisely defined energy, or set of energies. When they hit the surface of a solid, their identical impacts will launch identical, monochromatic phonons into it. These will skitter around the lattice, relaxing the dislocations they are tuned to. With luck, each dislocation as it relaxes will release its surplus energy as another phonon of that energy. A sort of phonon avalanche will spread through the lattice, relaxing it swiftly and completely. To test the process, the team will dissolve its products in nitric acid, and measure their heat of solution. Fully relaxed components, completely free of internal energy, will give out far less heat than the tense, stressed products of traditional metal-bashing.

Tuned heat will usher in a new era of engineering. Nuts, bolts, girders, panels, all will be freed from their hidden tensions. Relaxed and confident, they will at last accept loads up to their full strength. Brittle failure, fatigue and even rusting, all of which usually start at some highly stressed site, will vanish too. Lighter, stronger, more reliable designs will be possible. A portable tuned heater could even relax existing stress-burdened structures in situ. Old cars and bridges, and aircraft reaching the end of their fatigue lifetimes, will be swiftly rejuvenated. Released from their besetting worry, engineers will relax too. David Jones 